JOURNAL OF THE

AMERICAN MATHEMATICAL SOCIETY

Volume 14, Number 3, Pages 535-554

S 0894-0347(01)00368-X

Article electronically published on March 22, 2001

\title{
THE MCKAY CORRESPONDENCE AS AN EQUIVALENCE OF DERIVED CATEGORIES
}

\author{
TOM BRIDGELAND, ALASTAIR KING, AND MILES REID
}

To Andrei Tyurin on his 60th birthday

\section{INTRODUCTION}

The classical McKay correspondence relates representations of a finite subgroup $G \subset \mathrm{SL}(2, \mathbb{C})$ to the cohomology of the well-known minimal resolution of the Kleinian singularity $\mathbb{C}^{2} / G$. Gonzalez-Sprinberg and Verdier [10] interpreted the McKay correspondence as an isomorphism on $\mathrm{K}$ theory, observing that the representation ring of $G$ is equal to the $G$-equivariant $\mathrm{K}$ theory of $\mathbb{C}^{2}$. More precisely, they identify a basis of the $\mathrm{K}$ theory of the resolution consisting of the classes of certain tautological sheaves associated to the irreducible representations of $G$.

It is natural to ask what happens when $\mathbb{C}^{2}$ is replaced by an arbitrary nonsingular quasiprojective complex variety $M$ of dimension $n$ and $G$ by a finite group of automorphisms of $M$, with the property that the stabiliser subgroup of any point $x \in M$ acts on the tangent space $T_{x} M$ as a subgroup of $\operatorname{SL}\left(T_{x} M\right)$. Thus the canonical bundle $\omega_{M}$ is locally trivial as a $G$-sheaf, in the sense that every point of $M$ has a $G$-invariant open neighbourhood on which there is a nonvanishing $G$-invariant $n$-form. This implies that the quotient variety $X=M / G$ has only Gorenstein singularities.

A natural generalisation of the McKay correspondence would then be an isomorphism between the $G$-equivariant $\mathrm{K}$ theory of $M$ and the ordinary $\mathrm{K}$ theory of a crepant resolution $Y$ of $X$, that is, a resolution of singularities $\tau: Y \rightarrow X$ such that $\tau^{*}\left(\omega_{X}\right)=\omega_{Y}$. In the classical McKay case, the minimal resolution is crepant, but in higher dimensions crepant resolutions do not necessarily exist and, even when they do, they are not usually unique. However, it is now known that crepant resolutions of Gorenstein quotient singularities do exist in dimension $n=3$, through a case by case analysis of the local linear actions by Ito, Markushevich and Roan (see Roan [21] and references given there). In dimension $\geq 4$, even such quotient singularities only have crepant resolutions in rather special cases.

In this paper, we take the point of view that the appropriate way to formulate and prove the McKay correspondence on $\mathrm{K}$ theory is to lift it to an equivalence of derived categories. In itself, this is not a new observation and it turns out that it was actually known to Gonzalez-Sprinberg and Verdier (see also Reid [20. Conjecture 4.1]). Furthermore, if the resolution is constructed as a moduli space of

Received by the editors May 1, 2000 and, in revised form, November 1, 2000.

2000 Mathematics Subject Classification. Primary 14E15, 14J30; Secondary 18E30, 19L47.

Key words and phrases. Quotient singularities, McKay correspondence, derived categories. Earlier versions of this paper carried the additional title "Mukai implies McKay". 
$G$-equivariant objects on $M$, then the correspondence should be given by a FourierMukai transform determined by the universal object. This is the natural analogue of the classical statement that the tautological sheaves are a basis of the $\mathrm{K}$ theory. Both points of view are taken by Kapranov and Vasserot [15] in proving the derived category version of the classical two-dimensional McKay correspondence.

The new and remarkable feature is that, by using the derived category and Fourier-Mukai transforms and, in particular, techniques developed in [6] and [7], the process of proving the equivalence of derived categories - when it works - also yields a proof that the moduli space is a crepant resolution. More specifically, we will give a sufficient condition for a certain natural moduli space, namely Nakamura's GHilbert scheme, to be a crepant resolution for which the McKay correspondence holds as an equivalence of derived categories. This condition is automatically satisfied in dimensions 2 and 3 . Thus we simultaneously prove the existence of one crepant resolution of $X=M / G$ in three dimensions, without a case by case analysis, and verify the McKay correspondence for this resolution. We do not prove the McKay correspondence for an arbitrary crepant resolution although our methods should easily adapt to more general moduli spaces of $G$-sheaves on $M$, which may provide different crepant resolutions to the one considered here.

The $G$-Hilbert scheme $G$-Hilb $M$ was introduced by Nakamura as a good candidate for a crepant resolution of $M / G$. It parametrises $G$-clusters or 'scheme theoretic $G$-orbits' on $M$ : recall that a cluster $Z \subset M$ is a zero-dimensional subscheme, and a $G$-cluster is a $G$-invariant cluster whose global sections $\Gamma\left(\mathcal{O}_{Z}\right)$ are isomorphic to the regular representation $\mathbb{C}[G]$ of $G$. Clearly, a $G$-cluster has length $|G|$ and a free $G$-orbit is a $G$-cluster. There is a Hilbert-Chow morphism

$$
\tau: G \text {-Hilb } M \longrightarrow X,
$$

which, on closed points, sends a $G$-cluster to the orbit supporting it. Note that $\tau$ is a projective morphism, is onto and is birational on one component.

When $M=\mathbb{C}^{3}$ and $G \subset \mathrm{SL}(3, \mathbb{C})$ is Abelian, Nakamura [18 proved that $G$-Hilb $M$ is irreducible and is a crepant resolution of $X$ (compare also Reid [20] and Craw and Reid [8]). He conjectured that the same result holds for an arbitrary finite subgroup $G \subset \mathrm{SL}(3, \mathbb{C})$. Ito and Nakajima [12] observed that the construction of Gonzalez-Sprinberg and Verdier [10] is the $M=\mathbb{C}^{2}$ case of a natural correspondence between the equivariant $\mathrm{K}$ theory of $M$ and the ordinary $\mathrm{K}$ theory of $G$-Hilb $M$. They proved that this correspondence is an isomorphism when $M=\mathbb{C}^{3}$ and $G \subset \mathrm{SL}(3, \mathbb{C})$ is Abelian by constructing an explicit resolution of the diagonal in Beilinson style. Our approach via Fourier-Mukai transforms leaves this resolution of the diagonal implicit (it appears as the object $\mathcal{Q}$ of $\mathrm{D}(Y \times Y)$ in Section 6), and seems to give a more direct argument. Two of the main consequences of the results of this paper are that Nakamura's conjecture is true and that the natural correspondence on $\mathrm{K}$ theory is an isomorphism for all finite subgroups of $\mathrm{SL}(3, \mathbb{C})$.

Since it is not known whether $G$-Hilb $M$ is irreducible or even connected in general, we actually take as our initial candidate for a resolution $Y$ the irreducible component of $G$-Hilb $M$ containing the free $G$-orbits, that is, the component mapping birationally to $X$. The aim is to show that $Y$ is a crepant resolution, and to construct an equivalence between the derived categories $\mathrm{D}(Y)$ of coherent sheaves on $Y$ and $\mathrm{D}^{G}(M)$ of coherent $G$-sheaves on $M$. A more detailed analysis of the equivalence shows that $Y=G$-Hilb $M$ when $M$ has dimension 3 . 
We now describe the correspondence and our results in more detail. Let $M$ be a nonsingular quasiprojective complex variety of dimension $n$ and let $G \subset \operatorname{Aut}(M)$ be a finite group of automorphisms of $M$ such that $\omega_{M}$ is locally trivial as a $G$-sheaf. Put $X=M / G$ and let $Y \subset G$-Hilb $M$ be the irreducible component containing the free orbits, as described above. Write $\mathcal{Z}$ for the universal closed subscheme $\mathcal{Z} \subset Y \times M$ and $p$ and $q$ for its projections to $Y$ and $M$. There is a commutative diagram of schemes

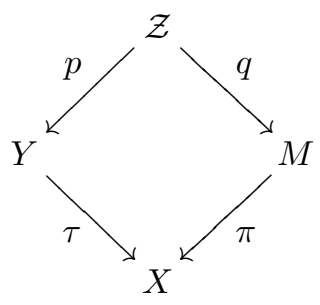

in which $q$ and $\tau$ are birational, $p$ and $\pi$ are finite, and $p$ is flat. Let $G$ act trivially on $Y$ and $X$, so that all morphisms in the diagram are equivariant.

Define the functor

$$
\Phi=\mathbf{R} q_{*} \circ p^{*}: \mathrm{D}(Y) \longrightarrow \mathrm{D}^{G}(M),
$$

where a sheaf $E$ on $Y$ is viewed as a $G$-sheaf by giving it the trivial action. Note that $p^{*}$ is already exact, so we do not need to write $\mathbf{L} p^{*}$. Our main result is the following.

Theorem 1.1. Suppose that the fibre product

$$
Y \times{ }_{X} Y=\left\{\left(y_{1}, y_{2}\right) \in Y \times Y \mid \tau\left(y_{1}\right)=\tau\left(y_{2}\right)\right\} \subset Y \times Y
$$

has dimension $\leq n+1$. Then $Y$ is a crepant resolution of $X$ and $\Phi$ is an equivalence of categories.

When $n \leq 3$ the condition of the theorem always holds because the exceptional locus of $Y \rightarrow X$ has dimension $\leq 2$. In this case we can also show that $G$-Hilb $M$ is irreducible, so we obtain

Theorem 1.2. Suppose $n \leq 3$. Then $G$-Hilb $M$ is irreducible and is a crepant resolution of $X$, and $\Phi$ is an equivalence of categories.

The condition of Theorem 1.1 also holds whenever $G$ preserves a complex symplectic form on $M$ and $Y$ is a crepant resolution of $X$, because such a resolution is symplectic and hence semi-small (see Verbitsky [24, Theorem 2.8] and compare Kaledin [14]).

Corollary 1.3. Suppose $M$ is a complex symplectic variety and $G$ acts by symplectic automorphisms. Assume that $Y$ is a crepant resolution of $X$. Then $\Phi$ is an equivalence of categories.

Note that the condition of Theorem 1.1 certainly fails in dimension $\geq 4$ whenever $Y \rightarrow X$ has an exceptional divisor over a point. This is to be expected since there are many examples of finite subgroups $G \subset \mathrm{SL}(4, \mathbb{C})$ for which the quotient singularity $\mathbb{C}^{4} / G$ has no crepant resolution and also examples where, although crepant resolutions do exist, $G$-Hilb $\left(\mathbb{C}^{4}\right)$ is not one. 
Conventions. We work throughout in the category of schemes over $\mathbb{C}$. A point of a scheme always means a closed point.

\section{Category theory}

This section contains some basic category theory, most of which is well known. The only nontrivial part is Section 2.6 where we state a condition for an exact functor between triangulated categories to be an equivalence.

2.1. Triangulated categories. A triangulated category is an additive category $\mathcal{A}$ equipped with a shift automorphism $T_{\mathcal{A}}: \mathcal{A} \rightarrow \mathcal{A}: a \mapsto a[1]$ and a collection of distinguished triangles

$$
a_{1} \stackrel{f_{1}}{\longrightarrow} a_{2} \stackrel{f_{2}}{\longrightarrow} a_{3} \stackrel{f_{3}}{\longrightarrow} a_{1}[1]
$$

of morphisms of $\mathcal{A}$ satisfying certain axioms (see Verdier [25]). We write $a[i]$ for $T_{\mathcal{A}}^{i}(a)$ and

$$
\operatorname{Hom}_{\mathcal{A}}^{i}\left(a_{1}, a_{2}\right)=\operatorname{Hom}_{\mathcal{A}}\left(a_{1}, a_{2}[i]\right) .
$$

A triangulated category $\mathcal{A}$ is trivial if every object is a zero object.

The principal example of a triangulated category is the derived category $\mathrm{D}(A)$ of an Abelian category $A$. An object of $\mathrm{D}(A)$ is a bounded complex of objects of $A$ up to quasi-isomorphism, the shift functor moves a complex to the left by one place and a distinguished triangle is the mapping cone of a morphism of complexes. In this case, for objects $a_{1}, a_{2} \in A$, one has $\operatorname{Hom}_{\mathrm{D}(A)}^{i}\left(a_{1}, a_{2}\right)=\operatorname{Ext}_{A}^{i}\left(a_{1}, a_{2}\right)$.

A functor $F: \mathcal{A} \rightarrow \mathcal{B}$ between triangulated categories is exact if it commutes with the shift automorphisms and takes distinguished triangles of $\mathcal{A}$ to distinguished triangles of $\mathcal{B}$. For example, derived functors between derived categories are exact.

2.2. Adjoint functors. Let $F: \mathcal{A} \rightarrow \mathcal{B}$ and $G: \mathcal{B} \rightarrow \mathcal{A}$ be functors. An adjunction for $(G, F)$ is a bifunctorial isomorphism

$$
\operatorname{Hom}_{\mathcal{A}}(G-,-) \cong \operatorname{Hom}_{\mathcal{B}}(-, F-) .
$$

In this case, we say that $G$ is left adjoint to $F$ or that $F$ is right adjoint to $G$. When it exists, a left or right adjoint to a given functor is unique up to isomorphism of functors. The adjoint of a composite functor is the composite of the adjoints. An adjunction determines and is determined by two natural transformations $\varepsilon: G \circ F \rightarrow$ $\operatorname{id}_{\mathcal{A}}$ and $\eta: \operatorname{id}_{\mathcal{B}} \rightarrow F \circ G$ that come from applying the adjunction to $1_{F a}$ and $1_{G b}$ respectively (see Mac Lane [16, IV.1] for more details).

The basic adjunctions we use in this paper are described in Section 3.1 below.

2.3. Fully faithful functors and equivalences. A functor $F: \mathcal{A} \rightarrow \mathcal{B}$ is fully faithful if for any pair of objects $a_{1}, a_{2}$ of $\mathcal{A}$, the map

$$
F: \operatorname{Hom}_{\mathcal{A}}\left(a_{1}, a_{2}\right) \rightarrow \operatorname{Hom}_{\mathcal{B}}\left(F a_{1}, F a_{2}\right)
$$

is an isomorphism. One should think of $F$ as an 'injective' functor. This is clearer when $F$ has a left adjoint $G: \mathcal{B} \rightarrow \mathcal{A}$ (or a right adjoint $H: \mathcal{B} \rightarrow \mathcal{A}$ ), in which case $F$ is fully faithful if and only if the natural transformation $G \circ F \rightarrow \operatorname{id}_{\mathcal{A}}$ (or $\operatorname{id}_{\mathcal{A}} \rightarrow H \circ F$ ) is an isomorphism.

A functor $F$ is an equivalence if there is an 'inverse' functor $G: \mathcal{B} \rightarrow \mathcal{A}$ such that $G \circ F \cong \operatorname{id}_{\mathcal{A}}$ and $F \circ G \cong \operatorname{id}_{\mathcal{B}}$. In this case $G$ is both a left and right adjoint to $F$ (see Mac Lane [16] IV.4]). In practice, we show that $F$ is an equivalence by writing 
down an adjoint (a priori, one-sided) and proving that it is an inverse. One simple example of this is the following.

Lemma 2.1. Let $\mathcal{A}$ and $\mathcal{B}$ be triangulated categories and $F: \mathcal{A} \rightarrow \mathcal{B}$ a fully faithful exact functor with a right adjoint $H: \mathcal{B} \rightarrow \mathcal{A}$. Then $F$ is an equivalence if and only if $H c \cong 0$ implies $c \cong 0$ for all objects $c \in \mathcal{B}$.

Proof. By assumption $\eta: \operatorname{id}_{\mathcal{A}} \rightarrow H \circ F$ is an isomorphism, so $F$ is an equivalence if and only if $\varepsilon: F \circ H \rightarrow \mathrm{id}_{\mathcal{B}}$ is an isomorphism. Thus the 'only if' part of the lemma is immediate, since $c \cong F H c$.

For the 'if' part, take any object $b \in \mathcal{B}$ and embed the natural adjunction map $\varepsilon_{b}$ in a triangle

$$
c \rightarrow F H b \stackrel{\varepsilon_{b}}{\longrightarrow} b \rightarrow c[1] .
$$

If we apply $H$ to this triangle, then $H\left(\varepsilon_{b}\right)$ is an isomorphism, because $\eta_{H b}$ is an isomorphism and $H\left(\varepsilon_{b}\right) \circ \eta_{H b}=1_{H b}$ ([16, IV.1, Theorem 1]). Hence $H c \cong 0$ and so $c \cong 0$ by hypothesis. Thus $\varepsilon_{b}$ is an isomorphism, as required.

One may understand this lemma in a broader context as follows. The triangle (11) shows that, when $F$ is fully faithful with right adjoint $H$, there is a 'semi-orthogonal' decomposition $\mathcal{B}=(\operatorname{Im} F, \operatorname{Ker} H)$, where

$$
\begin{aligned}
\operatorname{Im} F & =\{b \in \mathcal{B}: b \cong F a \text { for some } a \in \mathcal{A}\}, \\
\operatorname{Ker} H & =\{c \in \mathcal{B}: H c \cong 0\} .
\end{aligned}
$$

Since $F$ is fully faithful, the fact that $b \cong F a$ for some object $a \in \mathcal{A}$ necessarily means that $b \cong F H b$, so only zero objects are in both subcategories. The semiorthogonality condition also requires that $\operatorname{Hom}_{\mathcal{B}}(b, c)=0$ for all $b \in \operatorname{Im} F$ and $c \in \operatorname{Ker} H$, which is immediate from the adjunction. The lemma then has the very reasonable interpretation that if $\operatorname{Ker} H$ is trivial, then $\operatorname{Im} F=\mathcal{B}$ and $F$ is an equivalence. Note that if $G$ is a left adjoint for $F$, then there is a similar semiorthogonal decomposition on the other side $\mathcal{B}=(\operatorname{Ker} G, \operatorname{Im} F)$ and a corresponding version of the lemma. For more details on semi-orthogonal decompositions see Bondal [4].

2.4. Spanning classes and orthogonal decomposition. A spanning class for a triangulated category $\mathcal{A}$ is a subclass $\Omega$ of the objects of $\mathcal{A}$ such that for any object $a \in \mathcal{A}$

$$
\operatorname{Hom}_{\mathcal{A}}^{i}(a, \omega)=0 \quad \text { for all } \omega \in \Omega, i \in \mathbb{Z} \quad \text { implies } a \cong 0
$$

and

$$
\operatorname{Hom}_{\mathcal{A}}^{i}(\omega, a)=0 \quad \text { for all } \omega \in \Omega, i \in \mathbb{Z} \quad \text { implies } a \cong 0 .
$$

The following easy lemma is [6, Example 2.2].

Lemma 2.2. The set of skyscraper sheaves $\left\{\mathcal{O}_{x}: x \in X\right\}$ on a nonsingular projective variety $X$ is a spanning class for $\mathrm{D}(X)$.

A triangulated category $\mathcal{A}$ is decomposable as an orthogonal direct sum of two full subcategories $\mathcal{A}_{1}$ and $\mathcal{A}_{2}$ if every object of $\mathcal{A}$ is isomorphic to a direct sum $a_{1} \oplus a_{2}$ with $a_{j} \in \mathcal{A}_{j}$, and if

$$
\operatorname{Hom}_{\mathcal{A}}^{i}\left(a_{1}, a_{2}\right)=\operatorname{Hom}_{\mathcal{A}}^{i}\left(a_{2}, a_{1}\right)=0
$$


for any pair of objects $a_{j} \in \mathcal{A}_{j}$ and all integers $i$. The category $\mathcal{A}$ is indecomposable if for any such decomposition one of the two subcategories $\mathcal{A}_{i}$ is trivial. For example, if $X$ is a scheme, $\mathrm{D}(X)$ is indecomposable precisely when $X$ is connected. For more details see Bridgeland [6].

2.5. Serre functors. The properties of Serre duality on a nonsingular projective variety were abstracted by Bondal and Kapranov [5] into the notion of a Serre functor on a triangulated category. Let $\mathcal{A}$ be a triangulated category in which all the Hom sets are finite dimensional vector spaces. A Serre functor for $\mathcal{A}$ is an exact equivalence $S: \mathcal{A} \rightarrow \mathcal{A}$ inducing bifunctorial isomorphisms

$$
\operatorname{Hom}_{\mathcal{A}}(a, b) \rightarrow \operatorname{Hom}_{\mathcal{A}}(b, S(a))^{\vee} \quad \text { for all } a, b \in \mathcal{A}
$$

that satisfy a simple compatibility condition (see [5]). When a Serre functor exists, it is unique up to isomorphism of functors. We say that $\mathcal{A}$ has trivial Serre functor if for some integer $i$ the shift functor $[i]$ is a Serre functor for $\mathcal{A}$.

The main example is the bounded derived category of coherent sheaves $\mathrm{D}(X)$ on a nonsingular projective variety $X$, having the Serre functor

$$
S_{X}(-)=\left(-\otimes \omega_{X}\right)[\operatorname{dim} X]
$$

Thus $\mathrm{D}(X)$ has trivial Serre functor if and only if the canonical bundle of $X$ is trivial.

2.6. A criterion for equivalence. Let $F: \mathcal{A} \rightarrow \mathcal{B}$ be an exact functor between triangulated categories with Serre functors $S_{\mathcal{A}}$ and $S_{\mathcal{B}}$. Assume that $F$ has a left adjoint $G: \mathcal{B} \rightarrow \mathcal{A}$. Then $F$ also has a right adjoint $H=S_{\mathcal{A}} \circ G \circ S_{\mathcal{B}}^{-1}$.

Theorem 2.3. With assumptions as above, suppose also that there is a spanning class $\Omega$ for $\mathcal{A}$ such that

$$
F: \operatorname{Hom}_{\mathcal{A}}^{i}\left(\omega_{1}, \omega_{2}\right) \rightarrow \operatorname{Hom}_{\mathcal{B}}^{i}\left(F \omega_{1}, F \omega_{2}\right)
$$

is an isomorphism for all $i \in \mathbb{Z}$ and all $\omega_{1}, \omega_{2} \in \Omega$. Then $F$ is fully faithful.

Proof. See [6, Theorem 2.3].

Theorem 2.4. Suppose further that $\mathcal{A}$ is nontrivial, that $\mathcal{B}$ is indecomposable and that $F S_{\mathcal{A}}(\omega) \cong S_{\mathcal{B}} F(\omega)$ for all $\omega \in \Omega$. Then $F$ is an equivalence of categories.

Proof. Consider an object $b \in \mathcal{B}$. For any $\omega \in \Omega$ and $i \in \mathbb{Z}$ we have isomorphisms

$$
\begin{gathered}
\operatorname{Hom}_{\mathcal{A}}^{i}(\omega, G b)=\operatorname{Hom}_{\mathcal{A}}^{i}\left(G b, S_{\mathcal{A}} \omega\right)^{\vee}=\operatorname{Hom}_{\mathcal{B}}^{i}\left(b, F S_{\mathcal{A}} \omega\right)^{\vee} \\
=\operatorname{Hom}_{\mathcal{B}}^{i}\left(b, S_{\mathcal{B}} F \omega\right)^{\vee}=\operatorname{Hom}_{\mathcal{B}}^{i}(F \omega, b)=\operatorname{Hom}_{\mathcal{A}}^{i}(\omega, H b),
\end{gathered}
$$

using Serre duality and the adjunctions for $(G, F)$ and $(F, H)$. Since $\Omega$ is a spanning class we can conclude that $G b \cong 0$ precisely when $H b \cong 0$. Then the result follows from [6, Theorem 3.3].

The proof of Theorem 3.3 in [6] may be understood as follows. If Ker $H \subset$ $\operatorname{Ker} G$, then the semi-orthogonal decomposition described at the end of Section 2.3 becomes an orthogonal decomposition. Hence Ker $H$ must be trivial, because $\mathcal{B}$ is indecomposable and $\mathcal{A}$, and hence $\operatorname{Im} F$, is nontrivial. Thus $\operatorname{Im} F=\mathcal{B}$ and $F$ is an equivalence. 


\section{DERIVED CATEGORIES OF SHEAVES}

This section is concerned with various general properties of complexes of $\mathcal{O}_{X^{-}}$ modules on a scheme $X$. Note that all our schemes are of finite type over $\mathbb{C}$. Given a scheme $X$, define $\mathrm{D}^{\mathrm{qc}}(X)$ to be the (unbounded) derived category of the Abelian category $\mathrm{Q} \operatorname{coh}(X)$ of quasicoherent sheaves on $X$. Also define $\mathrm{D}(X)$ to be the full subcategory of $\mathrm{D}^{\mathrm{qc}}(X)$ consisting of complexes with bounded and coherent cohomology.

3.1. Geometric adjunctions. Here we describe three standard adjunctions that arise in algebraic geometry and are used frequently in what follows. For the first example, let $X$ be a scheme and $E \in \mathrm{D}(X)$ an object of finite homological dimension. Then the derived dual

$$
E^{\vee}=\mathbf{R} \mathcal{H} \operatorname{om}_{\mathcal{O}_{X}}\left(E, \mathcal{O}_{X}\right)
$$

also has finite homological dimension, and the functor $-\stackrel{\mathbf{L}}{\otimes} E$ is both left and right adjoint to the functor $-\stackrel{\mathbf{L}}{\otimes} E^{\vee}$.

For the second example take a morphism of schemes $f: X \rightarrow Y$. The functor

$$
\mathbf{R} f_{*}: \mathrm{D}^{\mathrm{qc}}(X) \longrightarrow \mathrm{D}^{\mathrm{qc}}(Y)
$$

has the left adjoint

$$
\mathbf{L} f^{*}: \mathrm{D}^{\mathrm{qc}}(Y) \longrightarrow \mathrm{D}^{\mathrm{qc}}(X) \text {. }
$$

If $f$ is proper, then $\mathbf{R} f_{*}$ takes $\mathrm{D}(X)$ into $\mathrm{D}(Y)$. If $f$ has finite Tor dimension (for example if $f$ is flat, or $Y$ is nonsingular), then $\mathbf{L} f^{*}$ takes $\mathrm{D}(Y)$ into $\mathrm{D}(X)$.

The third example is Grothendieck duality. Again take a morphism of schemes $f: X \rightarrow Y$. The functor $\mathbf{R} f_{*}$ has a right adjoint

$$
f^{!}: \mathrm{D}^{\mathrm{qc}}(Y) \longrightarrow \mathrm{D}^{\mathrm{qc}}(X)
$$

and moreover, if $f$ is proper and of finite Tor dimension, there is an isomorphism of functors

$$
f^{!}(-) \cong \mathbf{L} f^{*}(-) \stackrel{\mathbf{L}}{\otimes} f^{!}\left(\mathcal{O}_{Y}\right)
$$

Neeman [19] has recently given a completely formal proof of these statements in terms of the Brown representability theorem.

Let $X$ be a nonsingular projective variety of dimension $n$ and write $f: X \rightarrow Y=$ $\operatorname{Spec}(\mathbb{C})$ for the projection to a point. In this case $f^{!}\left(\mathcal{O}_{Y}\right)=\omega_{X}[n]$. The above statement of Grothendieck duality implies that the functor

$$
S_{X}(-)=\left(-\otimes \omega_{X}\right)[n]
$$

is a Serre functor on $\mathrm{D}(X)$.

3.2. Duality for quasiprojective schemes. In order to apply Grothendieck duality on quasiprojective schemes, we need to restrict attention to sheaves with compact support. The support of an object $E \in \mathrm{D}(X)$ is the locus of $X$ where $E$ is not exact, that is, the union of the supports of the cohomology sheaves of $E$. It is always a closed subset of $X$.

Given a scheme $X$, define the category $\mathrm{D}_{\mathrm{c}}(X)$ to be the full subcategory of $\mathrm{D}(X)$ consisting of complexes whose support is proper. Note that when $X$ itself is proper, $\mathrm{D}_{\mathrm{c}}(X)$ is just the usual derived category $\mathrm{D}(X)$. 
If $X$ is a quasiprojective variety and $i: X \hookrightarrow \bar{X}$ is some projective closure, then the functor $i_{*}$ embeds $\mathrm{D}_{\mathrm{c}}(X)$ as a full triangulated subcategory of $\mathrm{D}(\bar{X})$. By resolution of singularities, if $X$ is nonsingular we can assume that $\bar{X}$ is too. Then the Serre functor on $\mathrm{D}(\bar{X})$ restricts to give a Serre functor on $\mathrm{D}(X)$. Thus if $X$ is a nonsingular quasiprojective variety of dimension $n$, the category $\mathrm{D}_{c}(X)$ has a Serre functor given by (3).

The argument used to prove Lemma 2.2 is easily generalised to give the statement that the set of skyscraper sheaves $\left\{\mathcal{O}_{x}: x \in X\right\}$ on a nonsingular quasiprojective variety $X$ is a spanning class for $\mathrm{D}_{\mathrm{c}}(X)$.

3.3. Crepant resolutions. Let $X$ be a variety and $f: Y \rightarrow X$ a resolution of singularities. Given a point $x \in X$ define $\mathrm{D}_{x}(Y)$ to be the full subcategory of $\mathrm{D}_{\mathrm{c}}(Y)$ consisting of objects whose support is contained in the fibre $f^{-1}(x)$. We have the following categorical criterion for $f$ to be crepant.

Lemma 3.1. Assume that $X$ has rational singularities, that is, $\mathbf{R} f_{*} \mathcal{O}_{Y}=\mathcal{O}_{X}$. Suppose $\mathrm{D}_{x}(Y)$ has trivial Serre functor for each $x \in X$. Then $X$ is Gorenstein and $f: Y \rightarrow X$ is a crepant resolution.

Proof. The Serre functor on $\mathrm{D}_{x}(Y)$ is the restriction of the Serre functor on $\mathrm{D}_{\mathrm{c}}(Y)$. Hence, by Section 3.2 the condition implies that for each $x \in X$ the restriction of the functor $\left(-\otimes \omega_{Y}\right)$ to the category $\mathrm{D}_{x}(Y)$ is isomorphic to the identity. Since $\mathrm{D}_{x}(Y)$ contains the structure sheaves of all fattened neighbourhoods of the fibre $f^{-1}(x)$ this implies that the restriction of $\omega_{Y}$ to each formal fibre of $f$ is trivial. To get the result, we must show that $\omega_{X}$ is a line bundle and that $f^{*} \omega_{X}=\omega_{Y}$. Since $\omega_{X}=f_{*} \omega_{Y}$, this is achieved by the following lemma.

Lemma 3.2. Assume that $X$ has rational singularities. Then a line bundle $L$ on $Y$ is the pullback $f^{*} M$ of some line bundle $M$ on $X$ if and only if the restriction of $L$ to each formal fibre of $f$ is trivial. Moreover, when this holds, $M=f_{*} L$.

Proof. For each point $x \in X$, the formal fibre of $f$ over $x$ is the fibre product

$$
Y \times_{X} \operatorname{Spec}\left(\widehat{\mathcal{O}}_{X, x}\right)
$$

The restriction of the pullback of a line bundle from $X$ to each of these schemes is trivial because a line bundle has trivial formal stalks at points.

For the converse suppose that the restriction of $L$ to each of these formal fibres is trivial. The theorem on formal functions shows that the completions of the stalks of the sheaves $\mathbf{R}^{i} f_{*} \mathcal{O}_{Y}$ and $\mathbf{R}^{i} f_{*} L$ at any point $x \in X$ are isomorphic for each $i$. Since $X$ has rational singularities it follows that $\mathbf{R}^{i} f_{*} L=0$ for all $i>0$, and $M=f_{*} L$ is a line bundle on $X$.

Since $f^{*} M$ is torsion free, the natural adjunction map $\eta: f^{*} f_{*} L \rightarrow L$ is injective, so there is a short exact sequence

$$
0 \rightarrow f^{*} f_{*} L \stackrel{\eta}{\longrightarrow} L \rightarrow Q \rightarrow 0 .
$$

By the projection formula and the fact that $X$ has rational singularities,

$$
\mathbf{R}^{i} f_{*}\left(f^{*} M\right)=M \otimes \mathbf{R}^{i} f_{*} \mathcal{O}_{Y}=0 \quad \text { for all } i>0 .
$$

The fact that $\eta$ is the unit of the adjunction for $\left(f^{*}, f_{*}\right)$ implies that $f_{*} \eta$ has a left inverse, and in particular is surjective. Applying $f_{*}$ to (4) we conclude that $f_{*} Q=0$. 
Using the theorem on formal functions again, we can deduce that

$$
f_{*}\left(Q \otimes L^{-1}\right)=0 .
$$

In particular, $Q \otimes L^{-1}$ has no nonzero global sections. Tensoring (44) with $L^{-1}$ gives a contradiction unless $Q=0$. Hence $\eta$ is an isomorphism and we are done.

\section{4. $G$-SHEAVES}

Throughout this section $G$ is a finite group acting on a scheme $X$ (on the left) by automorphisms. As in the last section, all schemes are of finite type over $\mathbb{C}$. We list some results we need concerning the category of sheaves on $X$ equipped with a compatible $G$ action, or ' $G$-sheaves' for short. Since $G$ is finite, most of the proofs are trivial and are left to the reader. The main point is that natural constructions involving sheaves on $X$ are canonical, so commute with automorphisms of $X$.

4.1. Sheaves and functors. A $G$-sheaf $E$ on $X$ is a quasicoherent sheaf of $\mathcal{O}_{X^{-}}$ modules together with a lift of the $G$ action to $E$. More precisely, for each $g \in G$, there is a lift $\lambda_{g}^{E}: E \rightarrow g^{*} E$ satisfying $\lambda_{1}^{E}=\operatorname{id}_{E}$ and $\lambda_{h g}^{E}=g^{*}\left(\lambda_{h}^{E}\right) \circ \lambda_{g}^{E}$.

If $E$ and $F$ are $G$-sheaves, then there is a (right) action of $G$ on $\operatorname{Hom}_{X}(E, F)$ given by $\theta^{g}=\left(\lambda_{g}^{F}\right)^{-1} \circ g^{*} \theta \circ \lambda_{g}^{E}$ and the spaces $G$-Hom $X(E, F)$ of $G$-invariant maps give the morphisms in the Abelian categories $\mathrm{Qcoh}^{G}(X)$ and $\operatorname{Coh}^{G}(X)$ of $G$-sheaves.

The category $\operatorname{Qcoh}^{G}(X)$ has enough injectives (Grothendieck [9, Proposition $5.1 .2]$ ) so we may take $G$-equivariant injective resolutions. Since $G$ is finite, if $X$ is a quasiprojective scheme there is an ample invertible $G$-sheaf on $X$ and so we may also take $G$-equivariant locally free resolutions. The functors $G$-Ext ${ }_{X}^{i}(-,-)$ are the $G$-invariant parts of $\operatorname{Ext}_{X}^{i}(-,-)$ and are the derived functors of $G$-Hom $\operatorname{Hom}_{X}(-,-)$. Thus if $X$ is nonsingular of dimension $n$, so that $\mathrm{Qcoh}(X)$ has global dimension $n$, then the category $\mathrm{Qcoh}^{G}(X)$ also has global dimension $n$.

The local functors $\mathcal{H o m}$ and $\otimes$ are defined in the obvious way on $\mathrm{Qcoh}^{G}(X)$, as are pullback $f^{*}$ and pushforward $f_{*}$ for any $G$-equivariant morphism of schemes $f: X \rightarrow Y$. Thus, for example, $\lambda_{g}^{f^{*} E}=f^{*} \lambda_{g}^{E}$. Natural isomorphisms such as $\operatorname{Hom}_{X}\left(f^{*} E, F\right) \cong \operatorname{Hom}_{Y}\left(E, f_{*} F\right)$ are canonical, that is, commute with isomorphisms of the base, and hence are $G$-equivariant. Therefore they restrict to natural isomorphisms

$$
G-\operatorname{Hom}_{X}\left(f^{*} E, F\right) \cong G-\operatorname{Hom}_{Y}\left(E, f_{*} F\right) .
$$

In other words, $f^{*}$ and $f_{*}$ are also adjoint functors between the categories $\mathrm{Qcoh}^{G}(X)$ and $\operatorname{Qcoh}^{G}(Y)$.

Similarly, the natural isomorphisms implicit in the projection formula, flat base change, etc. are canonical and hence $G$-equivariant.

It seems worthwhile to single out the following point:

Lemma 4.1. Let $E$ and $F$ be $G$-sheaves on $X$. Then, as a representation of $G$, we have a direct sum decomposition

$$
\operatorname{Hom}_{X}(E, F)=\bigoplus_{i=0}^{k} G-\operatorname{Hom}_{X}\left(E \otimes \rho_{i}, F\right) \otimes \rho_{i}
$$

over the irreducible representations $\left\{\rho_{0}, \ldots, \rho_{k}\right\}$. 
Proof. The result amounts to showing that

$$
G-\operatorname{Hom}\left(\rho_{i}, \operatorname{Hom}_{X}(E, F)\right)=G-\operatorname{Hom}_{X}\left(E \otimes \rho_{i}, F\right) .
$$

Let $f: X \rightarrow Y=\operatorname{Spec}(\mathbb{C})$ be projection to a point, with $G$ acting trivially on $Y$ so that the map is equivariant. Then $\operatorname{Qcoh}^{G}(Y)$ is just the category of $\mathbb{C}[G]$ modules. Note that $\operatorname{Hom}_{X}(E, F)=f_{*} \mathcal{H}_{o m_{\mathcal{O}_{X}}}(E, F)$ and $f^{*} \rho_{i}=\mathcal{O}_{X} \otimes \rho_{i}$, so that the adjunction between $f^{*}$ and $f_{*}$ gives

$$
\begin{aligned}
G-\operatorname{Hom}_{Y}\left(\rho_{i}, f_{*} \mathcal{H o m}_{\mathcal{O}_{X}}(E, F)\right) & =G-\operatorname{Hom}_{X}\left(\mathcal{O}_{X} \otimes \rho_{i}, \mathcal{H o m}_{\mathcal{O}_{X}}(E, F)\right) \\
& =G-\operatorname{Hom}_{X}\left(E \otimes \rho_{i}, F\right),
\end{aligned}
$$

as required.

4.2. Trivial actions. If the group $G$ acts trivially on $X$, then any $G$-sheaf $E$ decomposes as a direct sum

$$
E=\bigoplus_{i} E_{i} \otimes \rho_{i}
$$

over the irreducible representations $\left\{\rho_{0}, \rho_{1}, \ldots, \rho_{k}\right\}$ of $G$ (where $\rho_{0}=\mathbf{1}$ is the trivial representation). The sheaves $E_{i}$ are just ordinary sheaves on $X$. Furthermore, $G$-Hom ${ }_{X}\left(E_{i} \otimes \rho_{i}, E_{j} \otimes \rho_{j}\right)=0$ for $i \neq j$. Thus the category $\operatorname{Qcoh}^{G}(X)$ decomposes as a direct sum $\bigoplus_{i} \operatorname{Qcoh}^{i}(X)$ and each summand is equivalent to $\mathrm{Qcoh}(X)$.

In particular, every $G$-sheaf $E$ has a fixed part $[E]^{G}$ and the functor

$$
[-]^{G}: \operatorname{Qcoh}{ }^{G}(X) \rightarrow \mathrm{Q} \operatorname{coh}(X)
$$

is the left and right adjoint to the functor

$$
-\otimes \rho_{0}: \mathrm{Q} \operatorname{coh}(X) \rightarrow \mathrm{Q} \operatorname{coh}^{G}(X),
$$

that is, 'let $G$ act trivially'. Both functors are exact.

4.3. Derived categories. The $G$-equivariant derived category $\mathrm{D}^{G}(X)$ is defined to be the full subcategory of the (unbounded) derived category of $\mathrm{Qcoh}^{G}(X)$ consisting of complexes with bounded and coherent cohomology.

The usual derived functors $\mathbf{R} \mathcal{H}$ om $, \stackrel{\mathbf{L}}{\otimes}, \mathbf{L} f^{*}$ and $\mathbf{R} f_{*}$ may be defined on the equivariant derived category, and, as for sheaves, the standard properties of adjunctions, projection formula and flat base change then hold because the implicit natural isomorphisms are sufficiently canonical.

One way to obtain an equivariant Grothendieck duality is to refer to Neeman's results [19]. Let $f: X \rightarrow Y$ be an equivariant morphism of schemes. The only thing to check is that equivariant pushdown $\mathbf{R} f_{*}$ commutes with small coproducts. This is proved exactly as in [19]. Then the functor $\mathbf{R} f_{*}$ has a right adjoint $f^{!}$, and (2) holds when $f$ is proper and of finite Tor dimension.

As in the nonequivariant case this implies that if $X$ is a nonsingular quasiprojective variety of dimension $n$, the full subcategory $\mathrm{D}_{\mathrm{c}}^{G}(X) \subset \mathrm{D}^{G}(X)$ consisting of objects with compact supports has a Serre functor

$$
S_{X}(-)=\left(-\otimes \omega_{X}\right)[n],
$$

where $\omega_{X}$ is the canonical bundle of $X$ with its induced $G$-structure. 
4.4. Indecomposability. If $G$ acts trivially on $X$, then the results of Section 4.2 show that $\mathrm{D}^{G}(X)$ decomposes as a direct sum of orthogonal subcategories indexed by the irreducible representations of $G$. More generally it is easy to see that $\mathrm{D}^{G}(X)$ is decomposable unless $G$ acts faithfully. We need the converse of this statement.

Lemma 4.2. Suppose a finite group $G$ acts faithfully on a quasiprojective variety $X$. Then $\mathrm{D}^{G}(X)$ is indecomposable.

Proof. Suppose that $\mathrm{D}^{G}(X)$ decomposes as an orthogonal direct sum of two subcategories $\mathcal{A}_{1}$ and $\mathcal{A}_{2}$. Any indecomposable object of $\mathrm{D}^{G}(X)$ lies in either $\mathcal{A}_{1}$ or $\mathcal{A}_{2}$ and

$$
\operatorname{Hom}_{D^{G}(X)}\left(a_{1}, a_{2}\right)=0 \quad \text { for all } a_{1} \in \mathcal{A}_{1}, a_{2} \in \mathcal{A}_{2} .
$$

Since the action of $G$ is faithful, the general orbit is free. Let $D=G \cdot x$ be a free orbit. Then $\mathcal{O}_{D}$ is indecomposable as a $G$-sheaf. Suppose without loss of generality that $\mathcal{O}_{D}$ lies in $\mathcal{A}_{1}$.

Let $\rho_{i}$ be an irreducible representation of $G$. The sheaf $\mathcal{O}_{X} \otimes \rho_{i}$ is indecomposable in $\mathrm{D}^{G}(X)$ and there exists an equivariant map $\mathcal{O}_{X} \otimes \rho_{i} \rightarrow \mathcal{O}_{D}$ so $\mathcal{O}_{X} \otimes \rho_{i}$ also lies in $\mathcal{A}_{1}$. Any indecomposable $G$-sheaf $E$ supported in dimension 0 has a section, so by Lemma 4.1 there is an equivariant map $\mathcal{O}_{X} \otimes \rho_{i} \rightarrow E$, and thus $E$ lies in $\mathcal{A}_{1}$.

Finally given an indecomposable $G$-sheaf $F$, take an orbit $G \cdot x$ contained in $\operatorname{Supp}(F)$ and let $i: G \cdot x \hookrightarrow X$ be the inclusion. Then $i_{*} i^{*}(F)$ is supported in dimension 0 and there is an equivariant map $F \rightarrow i_{*} i^{*}(F)$, so $F$ also lies in $\mathcal{A}_{1}$. Now $\mathcal{A}_{2}$ is orthogonal to all sheaves, hence is trivial.

\section{The intersection THEOREM}

Our proof that $G$-Hilb $M$ is nonsingular follows an idea developed in Bridgeland and Maciocia [7] for moduli spaces over K3 fibrations, and uses the following famous and difficult result of commutative algebra:

Theorem 5.1 (Intersection theorem). Let $(A, m)$ be a local $\mathbb{C}$-algebra of dimension d. Suppose that

$$
0 \rightarrow M_{s} \rightarrow M_{s-1} \rightarrow \cdots \rightarrow M_{0} \rightarrow 0
$$

is a complex of finitely generated free $A$-modules with each homology module $H_{i}\left(M_{\bullet}\right)$ an $A$-module of finite length. Then $s \geq d$. Moreover, if $s=d$ and $H_{0}\left(M_{\bullet}\right) \cong A / m$, then

$$
H_{i}\left(M_{\bullet}\right)=0 \text { for all } i \neq 0,
$$

and $A$ is regular.

The basic idea is as follows. Serre's criterion states that any finite length $A$ module has homological dimension $\geq d$ and that $A$ is regular precisely if there is a finite length $A$-module which has homological dimension exactly $d$. The intersection theorem gives corresponding statements for complexes of $A$-modules with finite length homology. As a rough slogan, "regularity is a property of the derived category". For the main part of the proof, see Roberts [22], [23]; for the final clause, see [7].

We may rephrase the intersection theorem using the language of support and homological dimension. If $X$ is a scheme and $E$ an object in $\mathrm{D}(X)$, then it is easy 
to check [7] that, for any point $x \in X$,

$$
x \in \operatorname{Supp} E \Longleftrightarrow \operatorname{Hom}_{\mathrm{D}(X)}^{i}\left(E, \mathcal{O}_{x}\right) \neq 0 \text { for some } i \in \mathbb{Z} \text {. }
$$

The homological dimension of a nonzero object $E \in \mathrm{D}(X)$, written hom $\operatorname{dim} E$, is the smallest nonnegative integer $s$ such that $E$ is isomorphic in $\mathrm{D}(X)$ to a complex of locally free sheaves on $X$ of length $s$ (that is, having $s+1$ terms). If no such integer exists we put hom $\operatorname{dim} E=\infty$. One can prove [7] that if $X$ is quasiprojective, and $n$ is a nonnegative integer, then hom $\operatorname{dim} E \leq n$ if and only if there is an integer $j$ such that for all points $x \in X$

$$
\operatorname{Hom}_{\mathrm{D}(X)}^{i}\left(E, \mathcal{O}_{x}\right)=0 \quad \text { unless } j \leq i \leq j+n .
$$

The two parts of Theorem 5.1 now become the following (cf. [7]).

Corollary 5.2. Let $X$ be a scheme and $E$ a nonzero object of $\mathrm{D}(X)$. Then

$$
\operatorname{codim}(\operatorname{Supp} E) \leq \operatorname{hom} \operatorname{dim} E \text {. }
$$

Corollary 5.3. Let $X$ be a scheme, and fix a point $x \in X$ of codimension $n$. Suppose that there is an object $E$ of $\mathrm{D}(X)$ such that for all points $z \in X$, and any integer $i$,

$$
\operatorname{Hom}_{\mathrm{D}(X)}^{i}\left(E, \mathcal{O}_{z}\right)=0 \text { unless } z=x \text { and } 0 \leq i \leq n .
$$

Suppose also that $H^{0}(E) \cong \mathcal{O}_{x}$. Then $X$ is nonsingular at $x$ and $E \cong \mathcal{O}_{x}$.

\section{The PROJECTIVE CASE}

The aim of this section is to prove Theorem 1.1 under the additional assumption that $M$ is projective. The quasiprojective case involves some further technical difficulties that we deal with in the next section. Take notation as in the Introduction. We break the proof up into seven steps.

SteP 1. Let $\pi_{Y}: Y \times M \rightarrow Y$ and $\pi_{M}: Y \times M \rightarrow M$ denote the projections. The functor $\Phi$ may be rewritten

$$
\Phi(-) \cong \mathbf{R} \pi_{M_{*}}\left(\mathcal{O}_{\mathcal{Z}} \otimes \pi_{Y}^{*}\left(-\otimes \rho_{0}\right)\right) .
$$

Note that $\mathcal{O}_{\mathcal{Z}}$ has finite homological dimension, because $\mathcal{Z}$ is flat over $Y$ and $M$ is nonsingular. Hence the derived dual $\mathcal{O}_{\mathcal{Z}}^{\vee}=\mathbf{R} \mathcal{H}$ om $_{\mathcal{O}_{Y \times M}}\left(\mathcal{O}_{\mathcal{Z}}, \mathcal{O}_{Y \times M}\right)$ also has finite homological dimension and we may define another functor $\Psi: \mathrm{D}^{G}(M) \rightarrow$ $\mathrm{D}(Y)$, by the formula

$$
\Psi(-)=\left[\mathbf{R} \pi_{Y_{*}}\left(\mathcal{P} \stackrel{\mathbf{L}}{\otimes} \pi_{M}^{*}(-)\right)\right]^{G},
$$

where $\mathcal{P}=\mathcal{O}_{\mathcal{Z}}^{\vee} \otimes \pi_{M}^{*}\left(\omega_{M}\right)[n]$.

Now $\Psi$ is left adjoint to $\Phi$ because of the three standard adjunctions described in Section 3.1. The functor $\pi_{M}^{*}$ is the left adjoint to $\mathbf{R} \pi_{M, *}$. The functor $-\otimes \mathcal{O}_{\mathcal{Z}}$ has the (left and right) adjoint $-\otimes \mathcal{O}_{\mathcal{Z}}^{\vee}$. Finally the functor $\pi_{Y}^{!}$has the left adjoint $\mathbf{R} \pi_{Y_{*}}$ and

$$
\pi_{Y}^{!}(-)=\pi_{Y}^{*}(-) \otimes \pi_{M}^{*}\left(\omega_{M}\right)[n] .
$$

SteP 2. The composite functor $\Psi \circ \Phi$ is given by

$$
\mathbf{R} \pi_{2 *}\left(\mathcal{Q} \stackrel{\mathbf{L}}{\otimes} \pi_{1}^{*}(-)\right),
$$


where $\pi_{1}$ and $\pi_{2}$ are the projections of $Y \times Y$ onto its factors, and $\mathcal{Q}$ is some object of $\mathrm{D}(Y \times Y)$. This is just composition of correspondences (see Mukai [17] Proposition 1.3]).

If $i_{y}:\{y\} \times Y \hookrightarrow Y \times Y$ is the closed embedding, then $\mathbf{L} i_{y}^{*}(\mathcal{Q})=\Psi \Phi \mathcal{O}_{y}$. For any pair of points $y_{1}, y_{2}$, one has $\mathcal{O}_{\left(y_{1}, y_{2}\right)}=i_{y_{1}, *} \mathcal{O}_{y_{2}}$ so that

(5) $\operatorname{Hom}_{\mathrm{D}(Y \times Y)}^{i}\left(\mathcal{Q}, \mathcal{O}_{\left(y_{1}, y_{2}\right)}\right)=\operatorname{Hom}_{\mathrm{D}(Y)}^{i}\left(\Psi \Phi \mathcal{O}_{y_{1}}, \mathcal{O}_{y_{2}}\right)=G-\operatorname{Ext}_{M}^{i}\left(\mathcal{O}_{Z_{y_{1}}}, \mathcal{O}_{Z_{y_{2}}}\right)$,

using the adjunctions for $\left(\mathbf{L} i^{*}, i_{*}\right)$ and $(\Psi, \Phi)$. Our first objective is to show that $\mathcal{Q}$ is supported on the diagonal $\Delta \subset Y \times Y$, or equivalently that the groups in (5) vanish unless $y_{1}=y_{2}$. When $n=3$ this plays the rôle of assumption (4.8) of Ito and Nakajima [12].

StEP 3 . Let $Z_{1}, Z_{2} \subset M$ be $G$-clusters. Then

$$
G-\operatorname{Hom}_{M}\left(\mathcal{O}_{Z_{1}}, \mathcal{O}_{Z_{2}}\right)= \begin{cases}\mathbb{C} & \text { if } Z_{1}=Z_{2}, \\ 0 & \text { otherwise. }\end{cases}
$$

To see this note that $\mathcal{O}_{Z}$ is generated as an $\mathcal{O}_{M}$-module by any nonzero constant section. But, since the global sections $\Gamma\left(\mathcal{O}_{Z}\right)$ form the regular representation of $G$, the constant sections are precisely the $G$-invariant sections. Hence any equivariant morphism maps a generator to a scalar multiple of a generator and so is determined by that scalar.

Let $y_{1}$ and $y_{2}$ be distinct points of $Y$. Serre duality, together with our assumption that $\omega_{M}$ is locally trivial as a $G$-sheaf, implies that

$$
G-\operatorname{Ext}_{M}^{n}\left(\mathcal{O}_{Z_{y_{1}}}, \mathcal{O}_{Z_{y_{2}}}\right)=G-\operatorname{Hom}_{M}\left(\mathcal{O}_{Z_{y_{2}}}, \mathcal{O}_{Z_{y_{1}}}\right)=0
$$

so that

$$
G \text {-Ext }{ }_{M}^{p}\left(\mathcal{O}_{Z_{y_{1}}}, \mathcal{O}_{Z_{y_{2}}}\right)=0 \quad \text { unless } 1 \leq p \leq n-1 .
$$

Hence $\mathcal{Q}$ restricted to $(Y \times Y) \backslash \Delta$ has homological dimension $\leq n-2$.

STEP 4. Now we apply the intersection theorem. If $y_{1}$ and $y_{2}$ are points of $Y$ such that $\tau\left(y_{1}\right) \neq \tau\left(y_{2}\right)$, then the corresponding clusters $Z_{y_{1}}$ and $Z_{y_{2}}$ are disjoint, so that the groups in (5) vanish. Thus the support of $\left.\mathcal{Q}\right|_{(Y \times Y) \backslash \Delta}$ is contained in the subscheme $Y \times{ }_{X} Y$. By assumption this has codimension $>n-2$ so Corollary 5.2 implies that

$$
\left.\mathcal{Q}\right|_{(Y \times Y) \backslash \Delta} \cong 0,
$$

that is, $\mathcal{Q}$ is supported on the diagonal.

SteP 5. Fix a point $y \in Y$, and put $E=\Psi \Phi\left(\mathcal{O}_{y}\right)$. We proved above that $E$ is supported at the point $y$. We claim that $H^{0}(E)=\mathcal{O}_{y}$. Note that Corollary $[5.3$ then implies that $Y$ is nonsingular at $y$ and $E \cong \mathcal{O}_{y}$.

To prove the claim, note that there is a canonical map $E \rightarrow \mathcal{O}_{y}$, so we obtain a triangle

$$
C \rightarrow E \rightarrow \mathcal{O}_{y} \rightarrow C[1]
$$

for some object $C$ of $\mathrm{D}(Y)$. Using the adjoint pair $(\Psi, \Phi)$, this gives a long exact sequence

$$
\begin{aligned}
\cdots \rightarrow & \operatorname{Hom}_{\mathrm{D}(Y)}^{0}\left(\mathcal{O}_{y}, \mathcal{O}_{y}\right) \rightarrow \operatorname{Hom}_{\mathrm{D}^{G}(M)}^{0}\left(\Phi \mathcal{O}_{y}, \Phi \mathcal{O}_{y}\right) \rightarrow \operatorname{Hom}_{\mathrm{D}(Y)}^{0}\left(C, \mathcal{O}_{y}\right) \\
& \rightarrow \operatorname{Hom}_{\mathrm{D}(Y)}^{1}\left(\mathcal{O}_{y}, \mathcal{O}_{y}\right) \stackrel{\varepsilon}{\longrightarrow} \operatorname{Hom}_{\mathrm{D}^{G}(M)}^{1}\left(\Phi \mathcal{O}_{y}, \Phi \mathcal{O}_{y}\right) \rightarrow \cdots
\end{aligned}
$$


The homomorphism $\varepsilon$ is the Kodaira-Spencer map for the family of clusters $\left\{\mathcal{O}_{Z_{y}}\right.$ : $y \in Y\}$ (Bridgeland [6] Lemma 4.4]). This is injective because $G$-Hilb $M$ is a fine moduli space for $G$-clusters on $M$. It follows that

$$
\operatorname{Hom}_{\mathrm{D}(Y)}^{i}\left(C, \mathcal{O}_{y}\right)=0 \text { for all } i \leq 0 .
$$

An easy spectral sequence argument (see [6, Example 2.2]) shows that $H^{0}(C)=0$. Taking cohomology sheaves of the above triangle gives $H^{0}(E)=\mathcal{O}_{y}$, which proves the claim.

STEP 6. We have now proved that $Y$ is nonsingular, and that for any pair of points $y_{1}, y_{2} \in Y$, the homomorphisms

$$
\Phi: \operatorname{Ext}_{Y}^{i}\left(\mathcal{O}_{y_{1}}, \mathcal{O}_{y_{2}}\right) \rightarrow G-\operatorname{Ext}_{M}^{i}\left(\mathcal{O}_{Z_{y_{1}}}, \mathcal{O}_{Z_{y_{2}}}\right)
$$

are isomorphisms. By assumption, the action of $G$ on $M$ is such that $\omega_{M}$ is trivial as a $G$-sheaf on an open neighbourhood of each orbit $G \cdot x \subset M$. This implies that

$$
\mathcal{O}_{Z_{y}} \otimes \omega_{M} \cong \mathcal{O}_{Z_{y}}
$$

in $\operatorname{Coh}^{G}(M)$, for each $y \in Y$. Applying Theorem 2.4 shows that $\Phi$ is an equivalence of categories.

SteP 7. It remains to show that $\tau: Y \rightarrow X$ is crepant. Take a point $x \in$ $X=M / G$. The equivalence $\Phi$ restricts to give an equivalence between the full subcategories $\mathrm{D}_{x}(Y) \subset \mathrm{D}(Y)$ and $\mathrm{D}_{x}^{G}(M) \subset \mathrm{D}^{G}(M)$ consisting of objects supported on the fibre $\tau^{-1}(x)$ and the orbit $\pi^{-1}(x)$ respectively.

The category $\mathrm{D}_{x}^{G}(M)$ has trivial Serre functor because $\omega_{M}$ is trivial as a $G$-sheaf on a neighbourhood of $\pi^{-1}(x)$. Thus $\mathrm{D}_{x}(Y)$ also has trivial Serre functor and Lemma 3.1 gives the result.

This completes the proof of Theorem 1.1 in the case that $Y$ is projective.

\section{The quasiprojective Case}

In this section we complete the proof of Theorem 1.1. Once again, take notation as in the Introduction. The problem with the argument of the last section is that when $M$ is not projective, Grothendieck duality in the form we need only applies to objects with compact support. To get round this we first take a projective closure $\bar{M}$ of $M$ and define adjoint functors as before. Then we restrict $\Phi$ to a functor

$$
\Phi_{\mathrm{c}}: \mathrm{D}_{\mathrm{c}}(Y) \longrightarrow \mathrm{D}_{\mathrm{c}}^{G}(M) \text {. }
$$

The argument of the last section carries through to show that $Y$ is nonsingular and crepant and that $\Phi_{\mathrm{c}}$ is an equivalence. It remains for us to show that $\Phi: \mathrm{D}(Y) \rightarrow$ $\mathrm{D}^{G}(M)$ is also an equivalence.

STEP 8. The functor $\Phi$ has a right adjoint

$$
\Upsilon(-)=\left[p_{*} \circ q^{!}(-)\right]^{G}=\left[\mathbf{R} \pi_{Y *}\left(\omega_{Z / M} \stackrel{\mathbf{L}}{\otimes} \pi_{M}^{*}(-)\right)\right]^{G} .
$$

As before, the composition $\Upsilon \circ \Phi$ is given by

$$
\mathbf{R} \pi_{2 *}\left(\mathcal{Q} \stackrel{\mathbf{L}}{\otimes} \pi_{1}^{*}(-)\right)
$$

where $\pi_{1}$ and $\pi_{2}$ are the projections of $Y \times Y$ onto its factors, and $\mathcal{Q}$ is some object of $\mathrm{D}(Y \times Y)$.

Since $\Phi_{\mathrm{c}}$ is an equivalence, $\Upsilon \Phi \mathcal{O}_{y}=\mathcal{O}_{y}$ for any point $y \in Y$, and it follows that $\mathcal{Q}$ is actually the pushforward of a line bundle $L$ on $Y$ to the diagonal in $Y \times Y$. 
The functor $\Upsilon \circ \Phi$ is then just twisting by $L$, and to show that $\Phi$ is fully faithful we must show that $L$ is trivial.

There is a morphism of functors $\varepsilon$ : id $\rightarrow \Upsilon \circ \Phi$, which for any point $y \in Y$ gives a commutative diagram

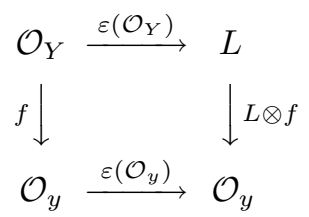

where $f$ is nonzero. Since $\varepsilon$ is an isomorphism on the subcategory $\mathrm{D}_{\mathrm{c}}(Y)$, the maps $\varepsilon\left(\mathcal{O}_{y}\right)$ are all isomorphisms, so the section $\varepsilon\left(\mathcal{O}_{Y}\right)$ is an isomorphism.

STEP 9. The fact that $\Phi$ is an equivalence follows from Lemma 2.1 once we show that

$$
\Upsilon(E) \cong 0 \Longrightarrow E \cong 0 \text { for any object } E \text { of } \mathrm{D}^{G}(M) .
$$

Suppose $\Upsilon(E) \cong 0$. Using the adjunction for $(\Phi, \Upsilon)$,

$$
\operatorname{Hom}_{\mathrm{D}^{G}(M)}^{i}(B, E)=0 \text { for all } i,
$$

whenever $B \cong \Phi(A)$ for some object $A \in \mathrm{D}(Y)$. In particular, this holds for any $B$ with compact support.

If $E$ is nonzero, let $D=G \cdot x$ be an orbit of $G$ contained in the support of $E$. Let $i: D \hookrightarrow M$ denote the inclusion, a projective equivariant morphism of schemes. Then the adjunction morphism $i_{*} i^{!}(E) \rightarrow E$ is nonzero, which gives a contradiction.

This completes the proof of Theorem 1.1

\section{NAKAmura's CONJECTURE}

Recall that in Theorem 1.1 we took the space $Y$ to be an irreducible component of $G$-Hilb $M$. Note that when $Y$ is nonsingular and $\Phi$ is an equivalence, $Y$ is actually a connected component. This is simply because for any point $y \in Y$, the bijection

$$
\Phi: \operatorname{Ext}_{Y}^{1}\left(\mathcal{O}_{y}, \mathcal{O}_{y}\right) \rightarrow G-\operatorname{Ext}_{M}^{1}\left(\mathcal{O}_{Z_{y}}, \mathcal{O}_{Z_{y}}\right)
$$

identifies the tangent space of $Y$ at $y$ with the tangent space of $G$-Hilb $M$ at $y$. In this section we wish to go further and prove that when $M$ has dimension 3, $G$-Hilb $M$ is in fact connected.

Proof of Nakamura's conjecture. Suppose for contradiction that there exists a $G$ cluster $Z \subset M$ not contained among the $\left\{Z_{y}: y \in Y\right\}$. Since $\Phi$ is an equivalence we can take an object $E \in \mathrm{D}_{\mathrm{c}}(Y)$ such that $\Phi(E)=\mathcal{O}_{Z}$. The argument of Section 6 Step 3, shows that for any point $y \in Y$

$$
\operatorname{Hom}_{\mathrm{D}(Y)}^{i}\left(E, \mathcal{O}_{y}\right)=G-\operatorname{Ext}_{M}^{i}\left(\mathcal{O}_{Z}, \mathcal{O}_{Z_{y}}\right)=0 \quad \text { unless } 1 \leq i \leq 2 .
$$

This implies that $E$ has homological dimension 1, or more precisely, that $E$ is quasi-isomorphic to a complex of locally free sheaves of the form

$$
0 \rightarrow L_{2} \stackrel{f}{\longrightarrow} L_{1} \rightarrow 0 .
$$

But $\mathcal{O}_{Z}$ is supported on some $G$-orbit in $M$, so $E$ is supported on a fibre of $Y$, and hence in codimension $\geq 1$. It follows that the complex (6) is exact on the left, so $E \cong$ coker $f[1]$. In particular $[E]=-[\operatorname{coker} f]$ in the Grothendieck group $K_{\mathrm{c}}(Y)$ of $\mathrm{D}_{\mathrm{c}}(Y)$. 
Let $y$ be a point of the fibre that is the support of $E$. By Lemma 8.1 below, $\left[\mathcal{O}_{Z_{y}}\right]=\left[\mathcal{O}_{Z}\right]$ in $K_{\mathrm{c}}^{G}(M)$, so that $\left[\mathcal{O}_{y}\right]=[E]$ in $K_{\mathrm{c}}(Y)$, since the equivalence $\Phi$ gives an isomorphism of Grothendieck groups.

Let $\bar{Y}$ be a nonsingular projective variety with an open inclusion $i: Y \hookrightarrow \bar{Y}$. The functor $i_{*}: \mathrm{D}_{\mathrm{c}}(Y) \rightarrow \mathrm{D}(\bar{Y})$ induces a map on $\mathrm{K}$ groups, so $[$ coker $f]=-\left[\mathcal{O}_{y}\right]$ in $K_{\mathrm{c}}(\bar{Y})$. But this contradicts Riemann-Roch, because if $L$ is a sufficiently ample line bundle on $\bar{Y}$, then $\chi(\operatorname{coker} f \otimes L)$ and $\chi\left(\mathcal{O}_{y} \otimes L\right)$ are both positive.

Lemma 8.1. If $Z_{1}$ and $Z_{2}$ are two $G$-clusters on $M$ supported on the same orbit, then the corresponding elements $\left[\mathcal{O}_{Z_{1}}\right]$ and $\left[\mathcal{O}_{Z_{2}}\right]$ in the Grothendieck group $K_{\mathrm{c}}^{G}(M)$ of $\mathrm{D}_{\mathrm{c}}^{G}(M)$ are equal.

Proof. We need to show that, as $G$-sheaves, $\mathcal{O}_{Z_{1}}$ and $\mathcal{O}_{Z_{2}}$ have composition series with the same simple factors. Suppose that they are both supported on the $G$-orbit $D=G \cdot x \subset M$ and let $H$ be the stabiliser subgroup of $x$ in $G$. The restriction functor is an equivalence of categories from finite length $G$-sheaves supported on $D$ to finite length $H$-sheaves supported at $x$. The reverse equivalence is the induction functor $\left(-\otimes_{\mathbb{C}[H]} \mathbb{C}[G]\right)$. Since the restriction of a $G$-cluster supported on $D$ is an $H$-cluster supported at $x$, it is sufficient to prove the result for $H$-clusters supported at $x$.

If $\left\{\rho_{0}, \ldots, \rho_{k}\right\}$ are the irreducible representations of $H$, then we claim that the simple $H$-sheaves supported at $x$ are precisely

$$
\left\{S_{i}=\mathcal{O}_{x} \otimes \rho_{i}: 0 \leq i \leq k\right\}
$$

These sheaves are certainly simple, since they are simple as $\mathbb{C}[H]$-modules. On the other hand, any $H$-sheaf $E$ supported at $x$ has a nonzero ordinary sheaf morphism $\mathcal{O}_{x} \rightarrow E$. By Lemma 4.1 there must be a nonzero $H$-sheaf morphism $S_{i} \rightarrow E$, for some $i$, and, if $E$ were simple, then this would have to be an isomorphism.

Thus a composition series as an $H$-sheaf is also a composition series as a $\mathbb{C}[H]$ module. Hence all $H$-clusters supported at $x$ have the same composition factors as $H$-sheaves, since as $\mathbb{C}[H]$-modules they are all the regular representation of $H$.

\section{K theoretic CONSEQUences of Equivalence}

In this section we put $M=\mathbb{C}^{n}$ and assume that the functor $\Phi$ is an equivalence of categories. This is always the case when $n \leq 3$. The main point is that such an equivalence of derived categories immediately gives an isomorphism of the corresponding Grothendieck groups.

9.1. Restricting to the exceptional fibres. Let $\mathrm{D}_{0}^{G}\left(\mathbb{C}^{n}\right)$ denote the full subcategory of $\mathrm{D}^{G}\left(\mathbb{C}^{n}\right)$ consisting of objects supported at the origin of $\mathbb{C}^{n}$. Similarly, let $\mathrm{D}_{0}(Y)$ denote the full subcategory of $\mathrm{D}(Y)$ consisting of objects supported on the subscheme $\tau^{-1}(\pi(0))$ of $Y$.

The equivalence $\Phi$ induces an equivalence

$$
\Phi_{0}: \mathrm{D}_{0}(Y) \rightarrow \mathrm{D}_{0}^{G}\left(\mathbb{C}^{n}\right)
$$


so we obtain a diagram

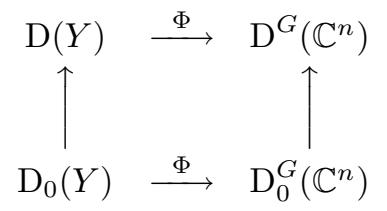

in which the vertical arrows are embeddings of categories.

Note that the Euler characteristic gives natural bilinear pairings between the top and bottom categories on either side; if $E$ and $F$ are objects of $\mathrm{D}^{G}\left(\mathbb{C}^{n}\right)$ and $\mathrm{D}_{0}^{G}\left(\mathbb{C}^{n}\right)$ respectively, then we can compute the sums

$$
\chi^{G}(E, F)=\sum_{i}(-1)^{i} \operatorname{dim} \operatorname{Hom}_{D^{G}\left(\mathbb{C}^{n}\right)}(E, F[i]),
$$

because the Hom spaces are finite dimensional (even over a quasiprojective variety) when $F$ has finite length cohomology sheaves. Similarly, we can compute the ordinary Euler character on the left. The fact that $\Phi$ is an equivalence of categories commuting with the shift functors immediately gives

$$
\chi^{G}(\Phi(A), \Phi(B))=\chi(A, B)
$$

for any objects $A$ of $\mathrm{D}(Y)$ and $B$ of $\mathrm{D}_{0}(Y)$.

9.2. Equivalence of $\mathbf{K}$ groups. Let $K(Y), K^{G}\left(\mathbb{C}^{n}\right), K_{0}(Y)$ and $K_{0}^{G}\left(\mathbb{C}^{n}\right)$ be the Grothendieck groups of the corresponding derived categories. The equivalences of categories from the last section immediately give isomorphisms of these groups. The following lemma is proved in the same way as in Gonzalez-Sprinberg and Verdier 10, Proposition 1.4].

Lemma 9.1. The maps that send a representation $\rho$ of $G$ to the $G$-sheaves $\rho \otimes \mathcal{O}_{\mathbb{C}^{n}}$ and $\rho \otimes \mathcal{O}_{0}$ on $\mathbb{C}^{n}$ give ring isomorphisms of the representation ring $R(G)$ with $K^{G}\left(\mathbb{C}^{n}\right)$ and $K_{0}^{G}\left(\mathbb{C}^{n}\right)$ respectively.

We obtain a diagram of groups

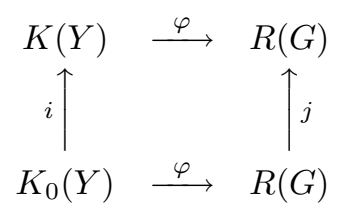

in which the horizontal maps are isomorphisms but the vertical maps are not. In fact, if $Q$ is the representation induced by the inclusion $G \subset \operatorname{SL}(n, \mathbb{C})$, then the map $j$ is multiplication by

$$
r=\sum_{i=0}^{n}(-1)^{i} \Lambda^{i} Q \in R(G) .
$$

This formula is obtained by considering a Koszul resolution of $\mathcal{O}_{0}$ on $M$, as in 10 . Proposition 1.4]. For example, in the case $n=2$ one has $r=2-Q$.

The bilinear forms of Section 9.1 descend to give pairings on the Grothendieck groups. These forms are nondegenerate because if $\left\{\rho_{0}, \ldots, \rho_{k}\right\}$ are the irreducible representations of $G$, then the corresponding bases

$$
\left\{\rho_{i} \otimes \mathcal{O}_{\mathbb{C}^{n}}\right\}_{i=0}^{k} \subset K^{G}\left(\mathbb{C}^{n}\right) \quad \text { and } \quad\left\{\rho_{i} \otimes \mathcal{O}_{0}\right\}_{i=0}^{k} \subset K_{0}^{G}\left(\mathbb{C}^{n}\right)
$$


are dual with respect to the pairing $\chi^{G}(-,-)$. Applying $\varphi^{-1}$ gives dual bases

$$
\left\{\mathcal{R}_{i}\right\}_{i=0}^{k} \subset K(Y) \text { and }\left\{\mathcal{S}_{i}\right\}_{i=0}^{k} \subset K_{0}(Y)
$$

as in Ito and Nakajima [12].

\section{TOPOLOGICAL K THEORY AND PHYSICS}

With notation as in the Introduction, suppose that $M$ is projective, and further that $Y$ is nonsingular and $\Phi: \mathrm{D}(Y) \rightarrow \mathrm{D}^{G}(M)$ is an equivalence. For example suppose that $n=2$ or 3 .

10.1. K theory and the orbifold Euler number. Let $\mathcal{K}^{*}(Y)$ denote the topological complex $\mathrm{K}$ theory of $Y$ and $\mathcal{K}_{G}^{*}(M)$ the $G$-equivariant topological $\mathrm{K}$ theory of $M$. There are natural forgetful maps

$$
\alpha_{Y}: K(Y) \rightarrow \mathcal{K}^{0}(Y) \quad \text { and } \quad \alpha_{M}: K^{G}(M) \rightarrow \mathcal{K}_{G}^{0}(M) .
$$

Since $\Phi$ and its inverse $\Psi$ are defined as correspondences, we may define correspondences

$$
\varphi: \mathcal{K}^{*}(Y) \rightarrow \mathcal{K}_{G}^{*}(M) \quad \text { and } \quad \psi: \mathcal{K}_{G}^{*}(M) \rightarrow \mathcal{K}^{*}(Y)
$$

compatible with the maps $\alpha$, using the functors $\otimes, f^{*}$ and $f_{*}$ (also written $f_{!}$) on topological K theory, which extend to equivariant K theory, as usual, because they are canonical. Note that the definition and compatibility of $f_{*}$ is nontrivial; see [1] for more details. But now the fact that $\Phi$ and $\Psi$ are mutually inverse implies that $\varphi$ and $\psi$ are mutually inverse, that is, we have a graded isomorphism

$$
\mathcal{K}^{*}(Y) \cong \mathcal{K}_{G}^{*}(M)
$$

Atiyah and Segal [2] observed that the physicists' orbifold Euler number of $M / G$ is the Euler characteristic of $\mathcal{K}_{G}^{*}(M)$, that is,

$$
e(M, G)=\operatorname{dim} \mathcal{K}_{G}^{0}(M) \otimes \mathbb{Q}-\operatorname{dim} \mathcal{K}_{G}^{1}(M) \otimes \mathbb{Q}
$$

On the other hand, since the Chern character gives a $\mathbb{Z} / 2$ graded isomorphism $\mathcal{K}^{*}(Y) \otimes \mathbb{Q} \cong H^{*}(Y, \mathbb{Q})$, the Euler characteristic of $\mathcal{K}^{*}(Y)$ is just the ordinary Euler number $e(Y)$ of $Y$. Hence the isomorphism (7) on topological $\mathrm{K}$ theory provides a natural explanation for the physicists' Euler number conjecture

$$
e(M, G)=e(Y) \text {. }
$$

This was verified in the case $n=2$ as a consequence of the original McKay correspondence (cf. 22). It was proved in the case $n=3$ by Roan 21 in the more general case of quasiprojective Gorenstein orbifolds, since the numerical statement reduces to the local linear case $M=\mathbb{C}^{3}, G \subset \mathrm{SL}(3, \mathbb{C})$.

10.2. An example: The Kummer surface. One of the first interesting cases of the isomorphism (77) is when $M$ is an Abelian surface (topologically, a 4-torus $\left.T^{4}\right), G=\mathbb{Z} / 2$ acting by the involution -1 and $Y$ is a K3 surface. In this case $Y$ is a nonsingular Kummer surface, having 16 disjoint -2-curves $C_{1}, \ldots, C_{16}$ coming from resolving the images in $M / G$ of the $16 G$-fixed points $x_{1}, \ldots, x_{16}$ in $M$. Write $V=\left\{x_{1}, \ldots, x_{16}\right\}$ for this fixed point set.

On the Abelian surface $M$ there are 32 flat line $G$-bundles, arising from a choice of $2 G$-actions on each of the 16 square roots of $\mathcal{O}_{M}$. Each such flat line $G$-bundle $L(\rho)$ is characterised by a map $\rho: V \rightarrow \mathbb{F}_{2}=\{0,1\}$ such that at a fixed point $x \in V$ the group $G$ acts on the fibre $L_{x}$ with weight $(-1)^{\rho(x)}$. Now the set $V$ naturally has 
the structure of an affine 4 -space over $\mathbb{F}_{2}$ and the maps $\rho$ that occur are precisely the affine linear maps, including the two constant maps corresponding to the two actions on $\mathcal{O}_{M}$.

On the other hand, on the K3 surface $Y$ one may consider the lattice $\mathbb{Z}^{V} \subset$ $H^{2}(Y, \mathbb{Z})$ spanned by $C_{1}, \ldots, C_{16}$ and the smallest primitive sublattice $\Lambda$ containing $\mathbb{Z}^{V}$. The elements of $\Lambda$ give precisely the rational linear combinations of the divisors $C_{1}, \ldots, C_{16}$ which are themselves divisors. It is easy to see that $\mathbb{Z}^{V} \subset \Lambda \subset\left(\frac{1}{2} \mathbb{Z}\right)^{V}$ and it can also be shown that the image of $\Lambda$ in the quotient $\left(\frac{1}{2} \mathbb{Z}\right)^{V} / \mathbb{Z}^{V} \cong \mathbb{F}_{2}^{V}$ consists of precisely the affine linear maps on $V$ (see Barth, Peters and Van de Ven [3, Chapter VIII, Proposition 5.5]).

We claim that under the correspondence $\Psi$, the flat line $G$-bundle $L(\rho)$ is taken to the line bundle $\mathcal{O}_{Y}(D(\rho))$, where

$$
D(\rho)=\frac{1}{2}\left(\sum_{i} \rho\left(x_{i}\right) C_{i}\right) .
$$

To check the claim note that $\mathcal{O}_{M}$ is taken to $\mathcal{O}_{Y}$, and that, in the local linear McKay correspondence for $\mathbb{C}^{2} /(\mathbb{Z} / 2)$, the irreducible representation of weight -1 is taken to the line bundle $\mathcal{O}\left(\frac{1}{2} C\right)$, dual to the -2 -curve $C$ resolving the singularity.

\section{ACKNOWLEDGEMENTS}

The first author would like to thank the ICTP, Trieste, and EPSRC for financial support whilst this paper was written.

\section{REFERENCES}

[1] M.F. Atiyah and F. Hirzebruch, The Riemann-Roch theorem for analytic embeddings, Topology 1 (1962) 151-166. MR 26:5593

[2] M.F. Atiyah and G. Segal, On equivariant Euler characteristics, J. Geom. Phys. 6 (1989) 671-677. MR 92c:19005

[3] W. Barth, C. Peters and A. Van de Ven, Compact Complex Surfaces, Ergeb. Math. 4, Springer, 1984. MR 86c:32026

[4] A. Bondal, Representation of associative algebras and coherent sheaves, Math. USSR Izv. 34 (1990) 23-41. MR 90i:14017

[5] A. Bondal and M. Kapranov, Representable functors, Serre functors, and mutations, Math. USSR Izv. 35 (1990) 519-541. MR 91b:14013

[6] T. Bridgeland, Equivalences of triangulated categories and Fourier-Mukai transforms, Bull. London Math. Soc. 31 (1999) 25-34. MR 99k:18014

[7] T. Bridgeland and A. Maciocia, Fourier-Mukai transforms for K3 and elliptic fibrations, preprint, math.AG 9908022.

[8] A. Craw and M. Reid, How to calculate $A$-Hilb $\mathbb{C}^{3}$, preprint, math.AG 9909085, 29 pp.

[9] A. Grothendieck, Sur quelques points d'algèbre homologique, Tôhoku Math. J. 9 (1957) 119-221. MR 21:1328

[10] G. Gonzalez-Sprinberg and J.-L. Verdier, Construction géométrique de la correspondance de McKay, Ann. Sci. École Norm. Sup. 16 (1983) 409-449. MR 85k:14019

[11] R. Hartshorne, Residues and Duality, Lect. Notes Math. 20, Springer (1966). MR 36:5145

[12] Y. Ito and H. Nakajima, McKay correspondence and Hilbert schemes in dimension three, preprint, math.AG 9803120; Topology 39 (2000) 1155-1191. CMP 2001:01

[13] Y. Ito and M. Reid, The McKay correspondence for finite subgroups of SL(3, $\mathbb{C})$, in Higher dimensional complex varieties (Trento, 1994), M. Andreatta et al., Eds., de Gruyter, 1996, pp. 221-240. MR 98i:14018

[14] D. Kaledin, The McKay correspondence for symplectic quotient singularities, preprint, math.AG 9907087.

[15] M. Kapranov and E. Vasserot, Kleinian singularities, derived categories and Hall algebras, preprint, math.AG 9812016; Math. Ann. 316 (2000) 565-576. CMP 2000:11 
[16] S. Mac Lane, Categories for the working mathematician, Graduate Texts in Math. 5, Springer, 1971. MR 50:7275

[17] S. Mukai, Duality between $\mathrm{D}(X)$ and $\mathrm{D}(\hat{X})$ with its application to Picard sheaves, Nagoya Math. J. 81 (1981) 153-175. MR 82f:14036

[18] I. Nakamura, Hilbert schemes of Abelian group orbits, to appear in J. Alg. Geom.

[19] A. Neeman, The Grothendieck duality theorem via Bousfield's techniques and Brown representability, J. Amer. Math. Soc. 9 (1996) 205-236. MR 96c:18006

[20] M. Reid, McKay correspondence, in Proc. of algebraic geometry symposium (Kinosaki, Nov 1996), T. Katsura (Ed.), 14-41, alg-geom 9702016.

[21] S.-S. Roan, Minimal resolutions of Gorenstein orbifolds in dimension 3, Topology 35 (1996) 489-508. MR 97c:14013

[22] P. Roberts, Intersection theorems, in Commutative algebra (Berkeley, 1987), MSRI Publ. 15, Springer, 1989, pp. 417-436. MR 90j:13024

[23] P. Roberts, Multiplicities and Chern classes in local algebra, CUP, 1998. MR 2001a:13029

[24] M. Verbitsky, Holomorphic symplectic geometry and orbifold singularities, preprint, math.AG 9903175; Asian J. Math. 4 (2000) 553-563. CMP 2001:05

[25] J.-L. Verdier, Des catégories dérivées des catégories abéliennes, Astérisque 239 (1996). MR 98c: 18007

Department of Mathematics and Statistics, University of Edinburgh, King's Buildings, Mayfield Road, Edinburgh EH9 3JZ, United Kingdom

E-mail address: tab@maths.ed.ac.uk

Department of Mathematical Sciences, University of Bath, Bath BA2 7AY, United KINGDOM

E-mail address: a.d.king@maths.bath.ac.uk

Math Institute, University of Warwick, Coventry CV4 7AL, United Kingdom

E-mail address: miles@maths.warwick.ac.uk 